\title{
Acute acalculous cholecystitis in a patient undergoing coronary artery bypass surgery
}

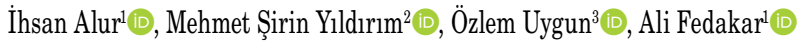 \\ ${ }^{1}$ Department of Cardiovascular Surgery, Private Hisar Hospital Intercontinental, Istanbul, Turkey \\ ${ }^{2}$ Department of Anesthesiology and Reanimation, Private Hisar Hospital Intercontinental, Istanbul, Turkey \\ ${ }^{3}$ Department of Radiology, Private Hisar Hospital Intercontinental, Istanbul, Turkey
}

Received: October 03, 2019 Accepted: November 26, 2019 Published online: March 16, 2020

\section{ABSTRACT}

Acute acalculous cholecystitis is a very rare complication of gastrointestinal system that can be seen after coronary artery bypass surgery. Early recognition of this clinical-pathological condition and initiation of treatment are very important and vital to prevent serious complications. In this article, we present a 67-year-old male patient of acalculous cholecystitis developing after coronary artery bypass grafting.

Keywords: Acalculous cholecystitis, coronary bypass, surgery.

Acute acalculous cholecystitis (AAC) is an acute inflammation of the gallbladder that develops without gallstones and has a multifactorial pathogenesis. ${ }^{[1]} \mathrm{It}$ generally occurs in male patients with an approximate male/female ratio of 2 to $3: 1$ and 60 years of age. ${ }^{[1]}$ In the etiology, many factors such as major surgery procedures, traumas, bacterial sepsis, viral infection, weakening/suppression of the immune system, collagen tissue diseases, and hyperalimentation have been charged while the cause has not been fully elucidated. ${ }^{[1]}$ Acute acalculous cholecystitis is most commonly associated with right upper quadrant pain, fever, and leukocytosis. ${ }^{[1]}$ Liver function tests, serum bilirubin and alkaline phosphatase levels increase. ${ }^{[1]}$ The most sensitive, specific, and low-cost imaging modality in the diagnosis is hepatobiliary ultrasonography (US). Dilatation of the gallbladder, presence of echogenic bile sludge, and thickening of the gallbladder wall of more than $3 \mathrm{~mm}$ are important sonographic findings. ${ }^{[1,2]}$ In this article, we report a case of AAC, which developed after coronary artery bypass grafting (CABG) and was successfully treated.

\section{CASE REPORT}

A 67-year-old male patient presented with atypical chest pain. He had a history of diabetes mellitus (DM), dyslipidemia, and hypertension (HT). On physical examination, his pulse rate was $84 / \mathrm{min}$, he had a blood pressure of $90 / 60 \mathrm{mmHg}$, and peripheral pulses were palpable. The electrocardiogram showed sinus rhythm. The patient's family history revealed that his father had died at the age of 48 due to myocardial infarction. Multiple coronary artery disease was diagnosed in coronary angiography. Subsequently, the patient underwent five-vessel CABG. The operation was routinely completed without complications. After the operation, the patient was admitted to the intensive care unit. On postoperative day three, the general condition of patient deteriorated, oxygen saturation decreased, and anxiety developed. Transthoracic echocardiography showed that left ventricular ejection fraction was $50 \%$, while pericardial effusion was $27 \mathrm{~mm}$ in the posterior wall and $25 \mathrm{~mm}$ in the right lateral wall. White blood cell count was $20,87010^{3} / \mu \mathrm{L}$ (normal range: $4.1610^{3} / \mu \mathrm{L}-10.2010^{3} / \mu \mathrm{L}$ ). Aspartate aminotransferase was $144 \mathrm{IU} / \mathrm{L}$ (normal range: 0-37), gamma-glutamyl transpeptidase was $190 \mathrm{IU} / \mathrm{L}$ (normal range: 7-60), alanine aminotransferase was 136 IU/L (normal range: 0-41), alkaline phosphatase was $74 \mathrm{IU} / \mathrm{L}$ (normal range: 40-129), and C-reactive protein was $1.79 \mathrm{mg} / \mathrm{dL}$ (normal range: 0-0.5). Because the liver enzymes of the patient were high,

Corresponding author: İhsan Alur, MD. Hisar Hospital Intercontinental, Kalp ve Damar Cerrahisi Bölümü, 34768 Ümraniye, İstanbul, Turkey.

Tel: +90 216 - 5241300 e-mail: alur_@@hotmail.com

\section{Citation:}

Alur İ, Yıldırım MŞ, Uygun 0̈, Fedakar A. Acute acalculous cholecystitis in a patient undergoing coronary artery bypass surgery. Cardiovasc Surg Int 2019;6(3):35-37. 

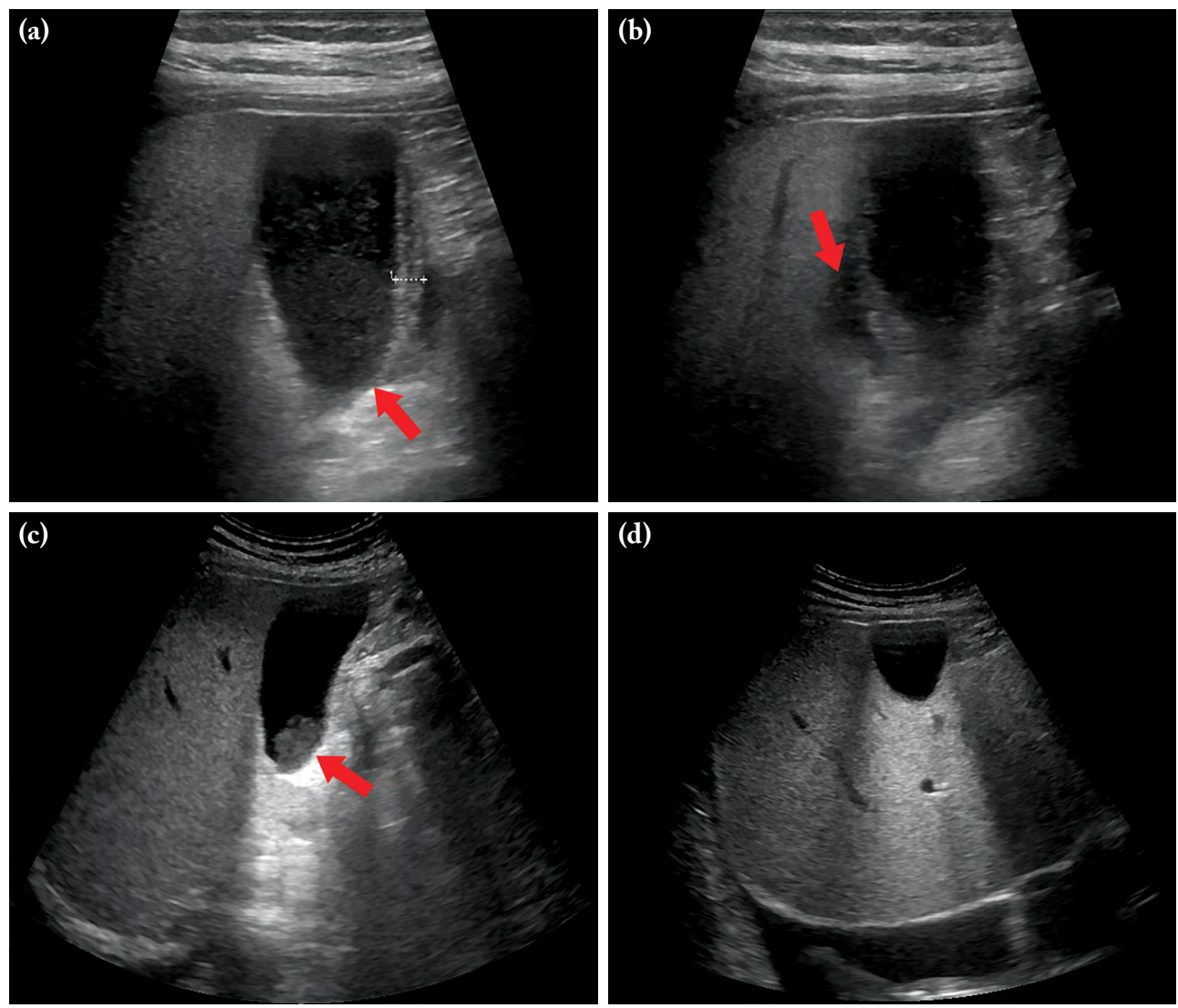

Figure 1. (a) Dense biliary sludge filling lumen of gallbladder (red arrow). (b) Fluid accumulation was observed in perihepatic area (red arrow). (c) Decreased biliary sludge filling lumen of gallbladder (red arrow). (d) Decreased perihepatic fluid and volume of gallbladder.

viral hepatitis markers were studied and found to be normal. Acute viral hepatitis was excluded in the differential diagnosis. Hepatobiliary US showed that liver was in normal shape/localization. Borders were regular. Parenchyma echogenicity was rough and Grade I hepatosteatosis was present. There was a slight increase in periportal echogenicity. There was no lesion that showed significant contour demarcation in the parenchyma. The width of the hepatic and portal veins was normal and the lumen was anechoic. The localization, morphology, and dimensions of the gallbladder were natural; the wall thickness was $6 \mathrm{~mm}$ and layered. Dense biliary sludge filling the lumen of the gallbladder was observed. Increased intensive internal echo and pericholecystic fluid were observed. There was no dilatation in intra-extrahepatic biliary tract and common bile duct. Fluid accumulation was observed in the perihepatic area (Figure 1a, b). A written informed consent was obtained from the patient.
Since there was no serious complication in our case, we followed the patient with medical treatment. Oral feeding was stopped for three days. The patient was fed with liver protective feeding solution. Liver enzymes returned to normal. Nausea-vomiting symptoms disappeared. One week later, the US findings were normal (Figure 1c, d). The patient was discharged on the postoperative $14^{\text {th }}$ day.

\section{DISCUSSION}

Acute acalculous cholecystitis is a rare but serious complication after CABG. Mostly, it is difficult to distinguish from AAC. This disease can progress rapidly. Early recognition of $\mathrm{AAC}$ and initiation of treatment are very important and crucial to prevent serious complications. Clinical symptoms and findings include right subcostal pain sensitivity, fever, leukocytosis, excessive sweating, weakness, loss of appetite, nausea, vomiting, and increase in liver 
function tests and bilirubin levels. ${ }^{[1]}$ In our case, most of these findings were present. Our patient did not develop fever due to use of antibiotics. There was no right subcostal pain due to postoperative analgesic. The absence of abdominal pain or lack of right subcostal sensitivity may be due to diabetic neuropathy. Acute acalculous cholecystitis has some lethal complications reported in the literature such as gangrene, perforation, peritonitis, pericholecystic abscess, empyema, and cholecystobiliary fistula. ${ }^{[2]}$ The most commonly used imaging modalities for diagnosis are hepatobiliary US or computed tomography (CT). These findings were reported in both hepatobiliary US and $\mathrm{CT}$ in our case.

The most commonly proposed mechanisms include bile stasis or ischemia. Bile stasis may occur after fasting, obstruction, chronic irritation or ileus (prolonged total parenteral nutrition), which may cause bile condensation. This may lead to a direct toxic effect on the gallbladder epithelium. ${ }^{[1]}$ The other mechanism is the development of organ ischemia due to many risks such as atherosclerosis, DM, HT and vasculitis syndromes. In one study, leukocyte migration and accumulation due to ischemia and reperfusion in the gallbladder wall, increased focal lymphatic permeability and dilatation due to local microvascular blockage, and gallbladder epithelial damage due to increased bile duct permeability have been reported. ${ }^{[3]}$ In our case, ischemia-related cholecystitis may have developed since DM, HT, and dyslipidemia (preoperative low-density lipoprotein cholesterol: $133 \mathrm{mg} / \mathrm{dL}$ and triglyceride: $364 \mathrm{mg} / \mathrm{dL}$ ) risk factors were present. In addition, the patient was a 67 -year-old male and was consistent with the literature. Therapeutic options of the AAC include medical conservative treatment, percutaneous transhepatic gallbladder drainage or surgical interventions such as cholecystectomy and cholecystostomy. ${ }^{[1]}$ The mortality rate was reported as $23-40 \%$ in patients with postoperative AAC. The risk of mortality is higher in patients older than 75 years and in diabetic patients. ${ }^{[2]}$

In conclusion, acute acalculous cholecystitis has a high mortality rate after coronary bypass surgery. Early intervention and treatment are life saving. We believe that this clinical entity may appear silent in diabetic postoperative patients and it should not be overlooked. We also believe that this phenomenon is stimulative and remarkable for cardiovascular surgery specialists.

\section{Declaration of conflicting interests}

The authors declared no conflicts of interest with respect to the authorship and/or publication of this article.

\section{Funding}

The authors received no financial support for the research and/or authorship of this article.

\section{REFERENCES}

1. Huffman JL, Schenker S. Acute acalculous cholecystitis: a review. Clin Gastroenterol Hepatol 2010;8:15-22.

2. Girgin S, Gedik E, Aldemir M, Yağmur Y. Current treatment and surgery of acute cholecystitis. Turkiye Klinikleri J Surg Med Sci 2007;3:44-9.

3. Laurila JJ, Ala-Kokko TI, Laurila PA, Saarnio J, Koivukangas V, Syrjälä $H$, et al. Histopathology of acute acalculous cholecystitis in critically ill patients. Histopathology 2005;47:485-92. 